2015-09-06

\title{
Accelerated Aging Test on Multiple XLPE MV Cables Simultaneously to Induce Water Trees
}

\author{
Shane Conlan \\ Technological University Dublin, shane.conlan@student.dit.ie \\ Jane Courtney \\ Technological University Dublin, jane.courtney@tudublin.ie \\ Tom Looby \\ ESB,Tom.Looby@esb.ie
}

Follow this and additional works at: https://arrow.tudublin.ie/engscheleart

Part of the Electrical and Electronics Commons, and the Power and Energy Commons

\section{Recommended Citation \\ This Conference Paper is brought to you for free and open access by the School of Electrical and Electronic Engineering at ARROW@TU Dublin. It has been accepted for inclusion in Conference papers by an authorized administrator of ARROW@TU Dublin. For more information, please contact arrow.admin@tudublin.ie, aisling.coyne@tudublin.ie,gerard.connolly@tudublin.ie.}

Conlan, S., Courtney, J. \& Looby, T. (2015) Accelerated Aging Test on Multiple XLPE MV Cables

Simultaneously to Induce Water Trees, 50th Universities Power Engineering Conference (UPEC), UK, 2015.

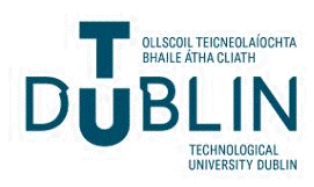




\section{Accelerated Aging Test on Multiple XLPE MV Cables Simultaneously to Induce Water Trees}

\author{
Shane Conlan \\ Dublin Institute of Technology, \\ Ireland \\ shanelukeconlan@gmail.com
}

\author{
Dr Jane Courtney \\ Dublin Institute of Technology, \\ Ireland \\ Jane.courtney@dit.ie
}

\author{
Tom Looby \\ ESB Networks, \\ Ireland \\ Tom.looby@esb.ie
}

\begin{abstract}
-
The objective of this paper is to develop an accelerated ageing test to induce water tree growth which is capable of testing up to ten $10 \mathrm{kV}$ cable samples simultaneously. An existing accelerated ageing method was used as a basis for this project. This method uses a high frequency power supply ( $3 \mathrm{kHz}$ ) to achieve a test duration of 14 days. Modifications were made to this method to allow for multiple samples to be tested simultaneously. A specially designed variable inductor was utilised in a parallel RLC resonance circuit. This resonance circuit limits the current draw from a high frequency high voltage transformer which allows for up to ten sample to undergo the test.

Water trees were found in a number of the samples tested. The maximum length and quantity of water trees in each sample was recorded and analysed. A ranking system was used to grade how each cable preformed during the test. The method of using the parallel resonance circuit to limit the current was successful. The accelerated ageing test worked as expected and water trees were detected in tested cable samples.
\end{abstract}

Index Terms-Accelerated, Ageing, Cables, Resonance, Medium, Trees, Voltage, Water, XLPE.

\section{Introduction}

Utilities companies in Ireland install approximately 3 million euro worth of medium voltage (MV) cable every year. From a financial and reliability point of view, these companies need to be certain, the lifespan of this investment is as outlined by the cable manufacturer. One of the factors which heavily contributes to the longevity of these cables is the quality of the polymer based insulation. The polymeric insulation that is used on the MV cables that these companies utilise is crosslinked polyethylene (XLPE).

XLPE is used as a dielectric by almost all cable manufactures today as it has excellent electrical insulating properties as well as mechanical properties such as strength, temperature rating and flexibility which make it an ideal insulator for electrical cables. However, polymer based insulators, including XLPE, have famously been prone to a phenomena known as water treeing.

"Water treeing is one of the major causes of premature failure of polyethylene cables" [1]. Water treeing is a process which breaks down the dielectric in electrical cables. The subject of water treeing has been widely studied in the past, especially in the 70's and 80's when the first polymeric MV cables began to fail due to these water trees. Quality of the insulation material has greatly improved as a result of these studies, leading to a reduction of water treeing in MV cable. Although this problem has been significantly reduced, it has not been eliminated and water trees can still be found in today's cables. Historically, water trees could only be identified by examining cables which has been in service for usually over 7 years. This was inconvenient, as sections of cable in service had to be removed for analysis. This motived the creation of the accelerated ageing test.

Accelerated ageing tests have been developed to test cables for their resistance to water tree growth in a relatively short period of time. CENELEC's HD 605 S2:2008 Method 7 [2] achieves this goal with an accelerated ageing test which lasts two years. This test produces results similar to that of a cable in service for 10-15 years. Hennuy et al [1] developed an accelerated ageing test which uses a high frequency, high voltage power supply, resulting in a test which only lasts for a duration of 14 days. An Irish utilities company has adopted this method in recent years to create their own $3 \mathrm{kHz}$ Accelerated Ageing Test. This method is limited to testing one $1 \mathrm{~m}$ cable sample over a 14 day period as multiple cables would cause the high frequency high voltage (HFHV) transformer to exceed its $\mathrm{kVA}$ rating. The aim of in this report is to build on this existing $3 \mathrm{kHz}$ accelerated ageing method to develop a test which can accelerate the age of 10 sample simultaneously. This would greatly reduce the overall time for testing cables against water tree growth. This method will overcome the loading issue by using a parallel resonance circuit to reduce the current flowing through the transformer. 


\section{DESIGN}

The problem with accelerated ageing multiple cable samples simultaneously, is the apparent power (VA) required to energise each sample at $3 \mathrm{kHz} 10 \mathrm{kV}$ is generally much greater than the VA rating of most commercially available high frequency, high voltage (HFHV) transformers. The HFHV transformer used in this paper had a rating of $1 \mathrm{kVA}$. This would mean that the maximum current draw from the transformer would have to be limited to $100 \mathrm{~mA}$

To achieve this, a resonating variable inductor was placed in parallel with the cable samples which would be used to create a resonance circuit and reducing the current drawn from the HFHV transformer

\section{TEST PROCEDURE}

The following procedure was undertaken as outlined by the utility company.

1. Sample Preparation and preconditioning: Test shall be performed on one meter of unused MV cable insulated with XLPE and having only the semi-conductive layers. With both ends of the cable exposed, the test sample shall be immersed in water for 500 hours at $55 \pm 5^{\circ} \mathrm{C}$ [2]. Tap water should be used with the addition of $0.3 \mathrm{~g}$ of $\mathrm{NaCl}$ per litre of water (sea water concentration).

2. Accelerated Ageing Test: The test set up shall be set up in accordance with the test parameters shown below.

3. Sample Analysis: Samples are subject to an examination for the presence of water trees following the accelerated aging test. Four, slices are located on each sample. These slices shall be taken from the samples using microtome cutting and be $600 \mu \mathrm{m}$ thick. Each slice is dyed in methylene blue in order to reveal water trees. Dyed slices are examined using stereo microscopy. The type, length and quantity of water trees are recorded if present.

Test Parameters: The following parameters shall be applied to the test.

- Voltage: $10 \mathrm{kV}$ [1]

- Frequency: $3 \mathrm{kHz}$ [12]

- Salt Water Solution: 0.3g ( NaCl) / L (H2O) [2]

- Ageing Water Temperature: $30 \pm 5^{\circ} \mathrm{C}[1]$

- $\quad$ Test Duration: 14 Days [1]

\section{Safety Precautions:}

The following safety instructions were carefully followed to avoid any hazardous situations.
- $\quad$ Arc resistant personal protective equipment (PPE) must be worn at all times.

- Calculations should be carried out to ensure the rating of any equipment will not be exceeded.

- The signal must remain at $3 \mathrm{kHz}$ at all times.

- The water solution should be at the same potential as earth.

- At the beginning of the test, start with a small voltage and slowly increase until the desired voltage level has been acquired.

- Monitor current levels and adjust the variable inductor until resonance has occurred.

- When the test is completed, slowly decrease the voltage supply to the samples. Disconnect and isolate any source of power. Earth busbar using earth clamps and a fiberglass earthing rod.

\section{RESULTS}

Following the accelerated aging test, a collection of results were obtained. These results compare the types of water trees found in each sample as well as the quantity of trees and the maximum length of each water tree.

Results from each cable sample were collected and analysed. Table I below, shows a breakdown of the results that were obtained from the stereo microscopy examination, i.e. water tree type, quantity of trees per $\mathrm{mm}^{3}$ and the maximum length of tree found in each sample.

TABLE I

TABulated Results For EACH CABle SAMPle

\begin{tabular}{|c|c|c|c|}
\hline Sample & Water Tree Type & Quantity $/ \mathrm{mm}^{3}$ & Max Length $(\mu \mathrm{m})$ \\
\hline \multirow{2}{*}{1} & Bow-tie trees & 0.041 & 247 \\
\hline & Vented trees & 0.092 & 58 \\
\hline \multirow{2}{*}{2} & Bow-tie trees & 0.002 & 45 \\
\hline & Vented trees & 0.023 & 104 \\
\hline \multirow{2}{*}{3} & Bow-tie trees & 0 & $\mathrm{~N} / \mathrm{A}$ \\
\hline & Vented trees & 0 & N/A \\
\hline \multirow{2}{*}{4} & Bow-tie trees & 0 & N/A \\
\hline & Vented trees & 0.039 & 30 \\
\hline \multirow[b]{2}{*}{5} & Bow-tie trees & 0.45 & 289 \\
\hline & Vented trees & 0.02 & 50 \\
\hline \multirow[b]{2}{*}{6} & Bow-tie trees & 0 & N/A \\
\hline & Vented trees & 0 & N/A \\
\hline \multirow[b]{2}{*}{7} & Bow-tie trees & 0 & N/A \\
\hline & Vented trees & 0.102 & 43 \\
\hline \multirow[b]{2}{*}{8} & Bow-tie trees & 0 & $\mathrm{~N} / \mathrm{A}$ \\
\hline & Vented trees & 0 & N/A \\
\hline \multirow[b]{2}{*}{9} & Bow-tie trees & 0.000678 & 200 \\
\hline & Vented trees & 0.092 & 50 \\
\hline \multirow[b]{2}{*}{10} & Bow-tie trees & 0 & N/A \\
\hline & Vented trees & 0 & N/A \\
\hline
\end{tabular}


From Table I above, it can be seen that samples 3, 6, 8 and 10 were found to have no trees of any kind whereas, there were at least one type of tree found in the remaining samples. Sample 1 exhibited the second largest bow-tie tree and vented tree. The largest vented tree was recorded in sample 2 which can be seen in the left image in Fig. 1 below. Sample 5 was found to have the largest bow-tie tree and the largest quantity of bow-tie trees. The lowest concentration of trees in a sample where tree were discovered was found to be sample 9 . Further analysis of these results are discussed in the next section.

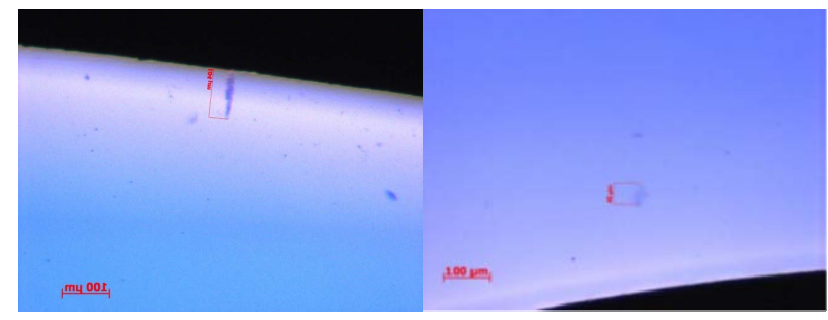

Fig. 1 Sample 2 Images-Vented Tree (Left), Bow-Tie Tree (Right)

\section{Bow-Tie Trees:}

Bow-tie trees are often caused by impurities or contaminants in the XLPE insulation. These types of trees are not considered as hazardous as vented trees as they tend to grow rapidly initially, but their growth rate also declines rapidly, resembling an inverse exponential curve. They can pose as substantial threat when the form close to a vented tree which may form into one large tree.

As shown in Fig. 3 below, it can be seen that only four cables were found with bow-tie trees.

\section{Vented Trees:}

Vented tree pose a much greater threat to a cables insulation. They form on the boundary of the XLPE and semi-conductive layers. They are considered much more dangerous as they grow more linearly. As a result, vented trees can break down the dielectric over time causing a path of low resistance for current to flow and lead to a complete failure of the cable.

As can be seen in Fig. 2, a total of 6 cables have developed water trees.

From Fig. 3 it can be seen that vented trees occur more often. This is likely due to imperfections on the surface of the semiconductive layers, where vented trees can originate from. However, the large quantity of bow-tie trees in sample 5 suggest strong contamination in the manufacturing process of this cable.

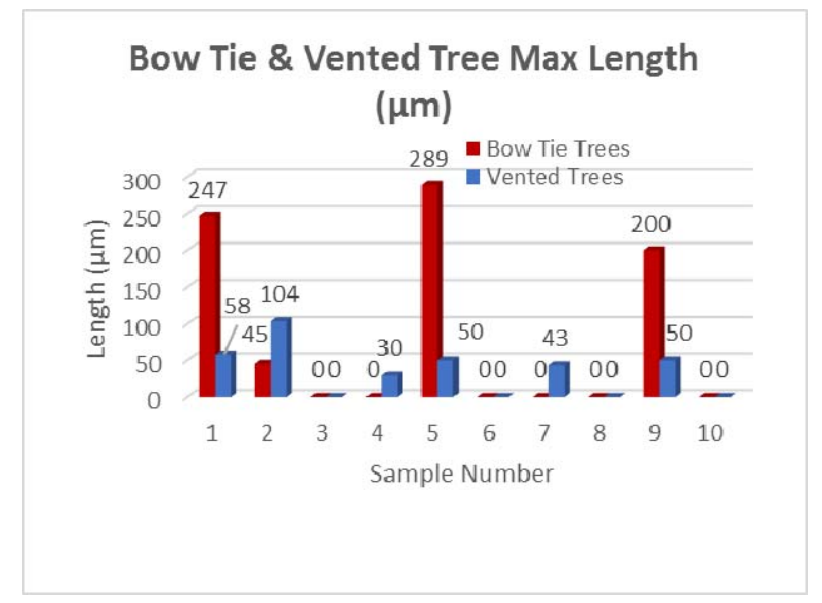

Fig. 2 Max Length: Bow-tie Vs Vented Trees

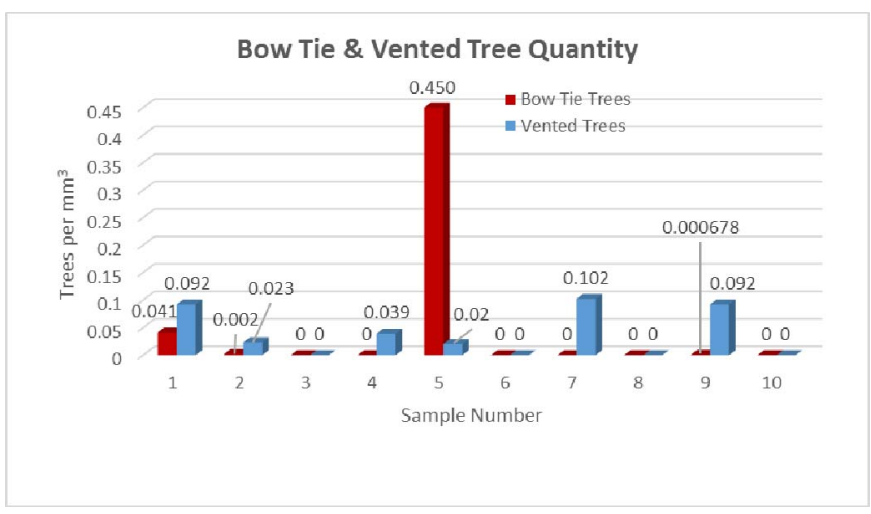

Fig. 3 Quantity: Bow-tie Vs Vented Trees

\section{Ranking \& Quality:}

To rank cable samples, a colour assignment was established which is based on previous test results [1]. The colour can change from green to yellow, orange, and red depending on the maximum length of the water trees detected by microscopic evaluation. Table II below shows how each cable is assigned a colour.

TABLE II

Colour Assignments Based On MaX Length Of Water Trees

\begin{tabular}{|l|c|c|}
\hline \multicolumn{3}{|c|}{ Quality Check Ranking System } \\
\hline Green & No VT's & No BT's \\
\hline Yellow & $\mathrm{VT}<100 \mu \mathrm{m}$ & $\mathrm{BT}<100 \mu \mathrm{m}$ \\
\hline Orange & $\mathrm{VT}<100-200 \mu \mathrm{m}$ & $\mathrm{BT}=100-200 \mu \mathrm{m}$ \\
\hline Red & $\mathrm{VT}>200 \mu \mathrm{m}$ & $\mathrm{BT}>200 \mu \mathrm{m}$ \\
\hline
\end{tabular}

The ranking system was applied to each cable sample tested to give an overall colour rank as shown in Table III. An overall colour rank of green is excellent, yellow is good, orange is poor and red is bad. 
TABLE III

COLOUR RANKING RESULTS

\begin{tabular}{|c|c|c|c|}
\hline $\begin{array}{c}\text { Sample } \\
\text { Number }\end{array}$ & $\begin{array}{c}\text { B.T. Max } \\
\text { Length }(\mu \mathrm{m})\end{array}$ & $\begin{array}{c}\text { V.T. Max } \\
\text { Length }(\mu \mathrm{m})\end{array}$ & $\begin{array}{c}\text { Overall Colour } \\
\text { Rank }\end{array}$ \\
\hline 1 & 247 & 58 & Orange \\
\hline 2 & 45 & 104 & Yellow \\
\hline 3 & 0 & 0 & Green \\
\hline 4 & 0 & 30 & Yellow \\
\hline 5 & 289 & 50 & Orange \\
\hline 6 & 0 & 0 & Green \\
\hline 7 & 200 & 43 & Orange \\
\hline 8 & 0 & 0 & Green \\
\hline 9 & 0 & 50 & Yellow \\
\hline 10 & 0 & 0 & Green \\
\hline
\end{tabular}

Cables 3, 6, 8 and 10 have obtained a green rank colour as no water trees of either type have been found in them. The yellow colour rank is applied to cables 2, 4 and 9, which indicates these cables performed well whereas cables 1,5 and 7 are considered poor cables as the fell into the orange rank.

\section{Comparison with Previous Accelerated Ageing Tests:}

Comparing these results with results from the previous $3 \mathrm{kHz}$ accelerated ageing test carried out by the utilities company, can give an indication on whether or not resistance to water tree growth has improved in recent years. Fig. 4 shows the results of a test carried out in 2013 .

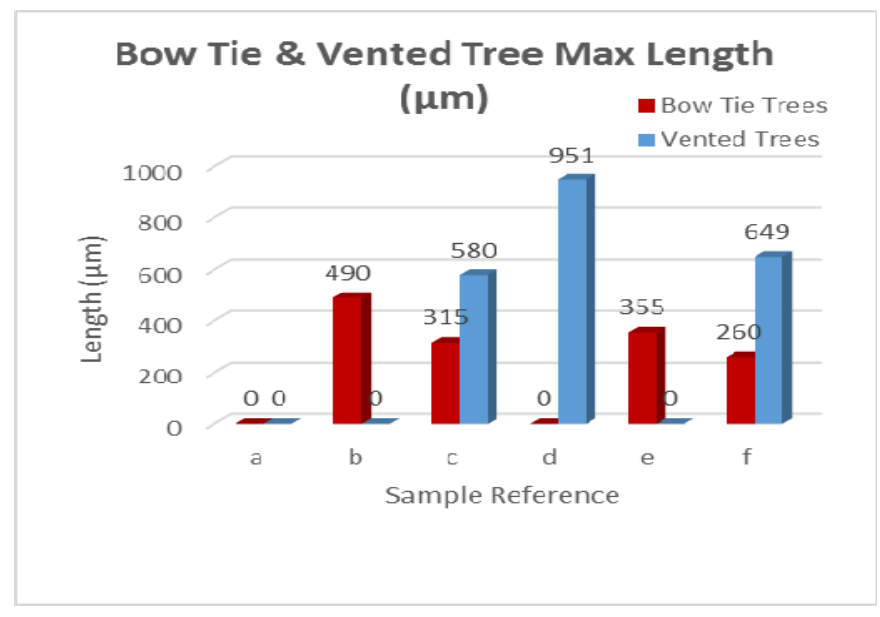

Fig. 4 Results for previous accelerated ageing tests. (2013)

The samples in Fig. 4 are different cables than were tested for this project. However, a trend of both large vented and large bow-tie tree can be observed. One of the most notable points to take from this graph is the length of the vented trees. These vented trees are up to 9 times larger the current vented trees. The average maximum length of tree Fig. 4 were found to be $300 \mu \mathrm{m}$ whereas the average maximum length of trees for this report were found to be $55.8 \mu \mathrm{m}$. This would suggest water tree resistance has improved overall especially in the case of vented trees

\section{CONCLUSION}

The use of the parallel resonance circuit proved to be an excellent solution to the problem of overloading the HFHV transformer. It was observed during the test start-up phase that by adjusting the remotely controlled variable inductor the current through the transformer windings was significantly reduced at resonance and began to rise either side of resonance.

The results from this test also show that the problem of water treeing in XLPE cables has not been eradicated as some would believe. This point is critical to electricity distributors who often feel research in this field does not deserve funding. Water treeing faults may be costing these company's tens of thousands annually in outage penalties and repair costs. By showing that water trees are still detectable in XLPE cable, some awareness may be raised to help reduce or eliminate water trees altogether.

There is strong evidence that would suggest the feedback that utility company gave the cable manufactures in relation to previous tests, has greatly improved. This can be seen as the average maximum length of trees has dropped from $300 \mu \mathrm{m}$ to $55.8 \mu \mathrm{m}$. This comparison demonstrates the usefulness and benefit of carrying out these tests. With the ability to now carry out 10 times more tests in the same time period, one can expect even greater improvements in the field of water treeing in XLPE cables in the near future.

The results from this test also show that the problem of water treeing in XLPE cables has not been eradicated as some would believe. This point is critical to electricity distributors who often feel research in this field does not deserve funding. Water treeing faults may be costing these company's tens of thousands annually in outage penalties and repair costs. By showing that water trees are still detectable in XLPE cable, some awareness may be raised to help reduce or eliminate water trees altogether.

\section{REFERENCES}

[1] B. Hennuy, Q. De Clerck, J. Marginet, D. Tenret, A. Francois and P. Leemans, "New Test Results with 3kHx Accelerated Growth of Water Trees in Medium Voltage XLPE Cables," in CIRED, Frankfurt, 2011.

[2] CENELEC, "Harmonisation Document HD 605 S2:2008 Electrical Cables: Additional Test Metods," ICS 29.060.20, 2008.

[3] J.-P. Crine and J. Jow, "Influence of frequency on water treeing in polyethylene," 2000 Conference on Electrical Insulation and Dialectric Phenomena, vol. 1, pp. 351$354,2000$. 